\title{
Fuzzy Mediation as a Dynamic Extension to Information Fusion
}

\author{
Giovanni Vincenti \\ Dept. of Computer and Information Sciences \\ Towson University \\ Towson, MD \\ giovanni.vincenti@gmail.com
}

\author{
Goran Trajkovski \\ Dept. of Information Technology \\ South University \\ Savannah, GA \\ gtrajkovski@southuniversity.edu
}

\begin{abstract}
This paper presents an innovative approach to the field of information fusion. Fuzzy mediation differentiates itself from other algorithms, as this approach is dynamic in nature. The experiments reported in this work analyze the interaction of two distinct controllers as they try to maneuver an artificial agent through a path. Fuzzy mediation functions as fusion engine to integrate the two inputs to produce a single output. Results show that fuzzy mediation is a valid method to mediate between two distinct controllers. The work reported in this article lays the foundation for the creation of an effective tool that uses positive feedback systems instead of negative ones to train human and non-human agents in the performance of control tasks.
\end{abstract}

Keywords: Fuzzy mediation; Information fusion; Shared control; Supervised learning; Collaborative learning.

\section{Introduction}

Technology plays a dominant role as each new product is designed and placed on the market. We can find high-end technological solutions in any types of objects, from fuzzy logic-powered rice cookers to the advanced fly-by-wire controls in jet planes. When technological solutions oversee the cooking of rice, we may or may not require much improvement. On the other hand, when we talk about an automated flight system that controls a passenger jetliner, we need to be sure that the concepts and solutions are well founded.

The fly-by-wire airplane idea is similar to the one of an automobile that operates with little input from the driver, or a joystick-operated heavy-duty machine that performs heavy jobs. The common denominator in these three examples is the existence of a computer mediating between signals from the operators and the actual sequence of actions taken by the machine in response to the operator. At times, the response of the machine depends on the operator's input, but there are times when the controls are autonomous responses to analyses of situations at hand.

Historically, the machine automation started by having the machines listen to either the input of the operator or to the one of a central controller. People realized that it was better to allow the operator and the electronic controller share control as much as possible. [1] points out that what we understand as shared control is not really shared. For example, in early stages of development, the machine used to supervise and control any part of the operations in flyby-wire airplanes, and now they oversee operations and still control flight, but as the conditions don't meet the standards of operations, the pilot is left alone. Therefore it is either the pilot controlling the plane on his/her own or the control system, and there is no interaction between the two. As we head for the future, we need a system that allows for greater interaction between the human operator and the digital one. Currently we have examples such as Caterpillar machines that allow operators to perform certain tasks only through automated systems [2]. Also, the two major airliner producers, Airbus and Boeing, are gearing towards advanced fly-by-wire technologies [3], as the auto industry is attempting to infuse automation concepts in their products [1].

Several aspects need to be addressed at this time, as the evolution of the interaction between machines and digital controllers increases. We need to 1) find a better way to mediate control in dual control systems, when two (or more) operators are controlling the same machine, and 2) then, replace one of the human operators with a digital one and investigate the interaction between the two entities using a mediation system (such as the proposed one in this paper). This can be done if we: 3 ) Create a framework for training using simulation and virtual reality to test-drive solutions and 4) implement these systems as a part of the actual operations of the machines under scrutiny. This list (1-4) outlines the goals that we have with this work.

Control is a concept that involves the interaction of multiple entities (by definition at least two). In a situation of control we can usually identify one subject who interacts directly with one object, directing the object's every move [4]. In environments where there is more than one operator controlling one object, we turn to the discipline of information fusion for solutions on joint control. Information fusion is defined as the process of taking multiple inputs and creating a single output [5]. As [5] states, many information fusion algorithms are biased in their operations. As they are designed, their operations are set and the fusion is carried out by simply running the 
algorithm. In most cases, the algorithms are pre-defined and static, and there is no adaptivity to the circumstances that influence the object. Adaptive information fusion systems, on the other hand, would offer an alternative where control can be effectively and truly shared between two (or more) operators.

A shared-control environment would allow for higherlevel applications to higher levels, in cases where supervised and collaborative learning is required, as in [6]. Supervised machine learning techniques represent a set of operations that "learn a task starting from a suite of examples" [7]. Information fusion would provide for supervised machine learning algorithms that learn from examples as the expert (operator) is operating (controlling) the machine. This approach represents a paradigmatical shift, as the learner becomes capable of interacting efficiently with the environment. It is an effective shift from the supervised learning domain to the collaborative learning one $[8,9]$. It has been noted, that many proposed solutions promote individual, instead of true collaborative learning [10]. [11] explored interaction between humans and computing when learning is performed by means of an apprentice model; however their focus is on the classification of items.

Another relevant concept to this paper is the concept of agent, as they attempt to solve issues of coordination, cooperation and learning [12]. Multiple agents' performance in an unknown environment is discussed by [13], where online collaborative learning is applied to several agents, facilitating the learning process itself.

\section{Fuzzy Mediation}

The concept of fuzzy mediation is an innovation that brings to the static world of information fusion a dynamic component that extends greatly the possibility of applications of this field of computing [14].

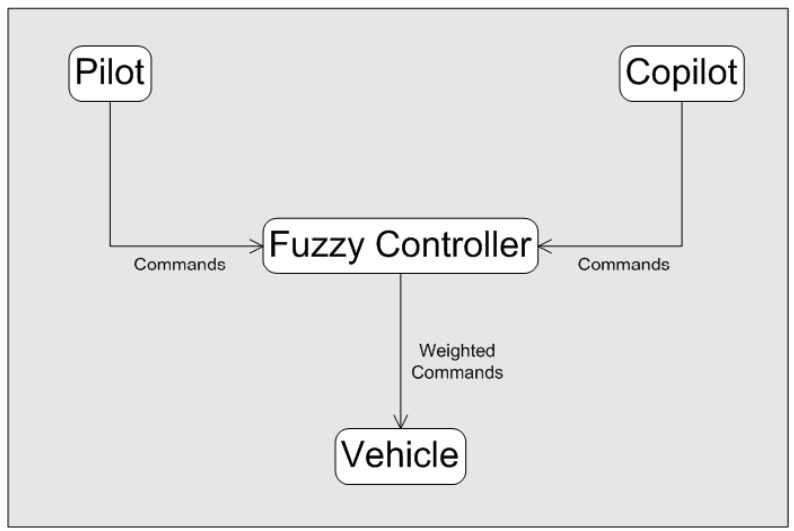

Figure 1. Typical scenario for fuzzy mediation

Fuzzy mediation aims to solve problems that are innate with the concept of shared control among multiple agents. The birth of this concept was set in a training environment where two agents are interacting. Both agents can be human, non-human or a mix of the two. In the typical scenario, depicted in Figure 1, the first controller functions as a pilot, or expert user, and the second controller functions as a copilot, of novice user. It is our assumption for this work that the expert controller is the one that performs actions as expected, and the novice controller is at an early phase of learning the tasks.

As it would be impossible for a vehicle to be controlled simultaneously by two different controllers, we looked at concepts of information fusion for a solution. The fuzzy controller performs three distinct operations. The first is the analysis of the inputs to determine the closeness of control; it then performs a revision of the weight of control between the expert and the novice controller; finally it computes the value of the single output.

\subsection{Analysis of the inputs}

Cantorian set theory leaves little room for gray areas; a room's temperature can be classified as hot, medium or cold. This system does not take into consideration the possibility of the same room being perceived as comfortable or chilly by two different persons. Fuzzy sets provide a solution that takes into consideration values that fall within multiple sets [15]. These kinds of sets can be utilized within the field of information fusion applied to a situation of multiple controllers trying to interact with a vehicle by a means of comparison.

The analysis of the inputs coming from the two controllers aims at understanding the distance between the values. The input coming from the expert user is mapped to the center of the range $[-10,10]$, as shown in Figure 2.

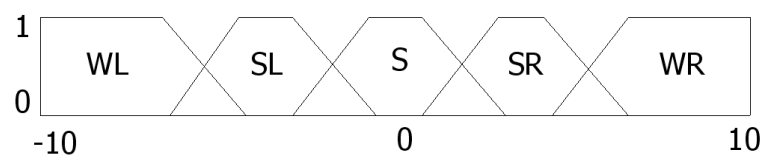

Figure 2. Breakdown of the sets used for comparing controller inputs

The range of $[-10,10]$ represents the highest possible deviation between the input of the expert and the one of the novice. After the input of the expert becomes the center of this domain, we calculate the difference between the value of the original input of the expert and the one of the novice. This value is then also mapped to the domain shown above.

When the distance between the inputs is mapped, it will fall within one or two sets that span over the range, as shown in Figure 2. The five sets we deal with are: WL (Wide deviation to the left), SL (Slight deviation to the left), S (Similar), SR (Slight deviation to the right), and WR (Wide deviation to the right). In fuzzy set theory, the value can belong to a set with a certain degree of belonging. Such degree of belonging is calculated by a membership function. In our case, we use a simple membership function, also shown in Figure 2. 
The application of a linguistic modifier to the deviation between the controller inputs also keeps in consideration the degree of belonging of the difference to the different sets. It is important to note that a value may fall completely (degree of belonging $=1$ ) within one set, or the value may belong mostly (.80) to the set of deviations deemed as Similar (S), and partly (.20) to the set of Slight deviations to the left (SL).

\subsection{Revision of the weight of control}

Fuzzy mediation sees the fusion of the inputs of the expert and the novice as a balance between the two. The more the novice performs similarly to the expert, the more control will shift in favor of the second controller. Likewise, the more the control of the novice differs from the one of the expert, the more control will shift back towards the expert.

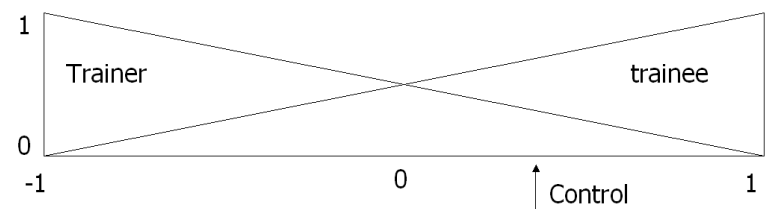

Figure 3. Fuzzy sets that regulate the balance of control between the expert and the novice controller

Given this preamble, this second part of the fuzzy mediation algorithm analyzes the linguistic modifiers applied to the deviation of the inputs during the first phase. Control is mapped to the range of $[-1,1]$, where a control weight of -1 identifies a control fully in the hands of the expert, a value of 1 instead refers to control managed by the novice. Figure 3 shows the visualization of this concept. As we apply the concept of fuzzy sets to this section of the algorithm, a value in the range $(-1,1)$ identifies a control that is mixed in a certain proportion between the expert and the novice. The arrow in Figure 3 shows a possible weight of the mediation of control between the trainer and the trainee. At the beginning of the simulation the weight has a value of -1 .

When the classification of the distance between inputs is analyzed, there are several actions that can be taken. If the inputs are classified as similar, control is given more to the novice. If the deviation between inputs is slight, then control stays unvaried. If instead the deviation is wide, more control is given to the novice. The shifting of the weight from one controller to the next occurs in a linear fashion, with increments or decrements of 0.2 points on the range $[-1,1]$ presented earlier. In the case of a distance between inputs that belongs to two sets, then we will multiply the degree of belonging to each of the sets to the action associated with that particular set. Using the example given earlier, a value that belongs to the set $\mathrm{S}$ with membership 0.8 will receive an increase in control of 0.2 (the standard increment) multiplied by the membership value, which means an increase of the weight of control of 0.16 . The same value also belongs to the set SL with membership 0.2. The action associated with a deviation that is classified as slight is a movement of 0 of the balance between controllers, so the action for this set is calculated by multiplying 0.2 , the membership value, to 0 . The addition of these values, 0.16 and 0 , shows the overall shift in control, which is of 0.16 in favor of the trainee.

\subsection{Calculation of the single output}

After the weight of control is updated, we need to calculate a value that will serve as a single input stemming from the original inputs of the two controllers. For this computation we need to refer to the original values. Equation 1 regulates the third section of this algorithm.

$$
\mathrm{MO}=\mu \mathrm{T} * \mathrm{EI}+\mu \mathrm{t} * \mathrm{NI}
$$

where MO symbolizes the mediated output, $\mu \mathrm{T}$ refers to the membership value of the weight of control to the Trainer set (Expert) and $\mu \mathrm{t}$ refers to the membership value of the weight of control to the trainee set (Novice). The inputs of the two controllers are represented by EI for the Expert's input and NI for the Novice's.

In the case of a driving simulator, we may have an expert applying a turn of 15 degrees to the right and a novice applying a turn of 25 degrees. If the weight of control has a value of -1 , the mediated output will have a value of 15 degrees to the right. Likewise, if the weight of control has a weight of 1 , the mediated output will be of 25 degrees to the right. If the weight is anywhere in between, for example $\mu \mathrm{T}=0.5$ and $\mu \mathrm{t}=0.5$, the mediated output will be of a 20 degree turn to the right.

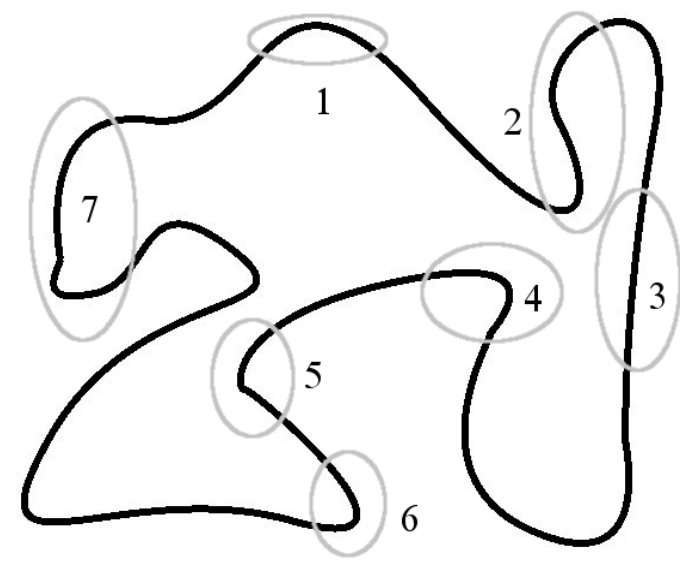

Figure 4. Path of the simulation

\section{Experimental setup}

The environment used for these experiments is a simple agent that follows a line. Figure 4 shows the pattern that was used. The pattern contains only the white background and the black line. The areas that have been highlighted show the sections of most interest. Section 1 was created to see the behavior of the agent in a mild turn to the right; section 2 instead simulates a sharp turn to the left followed 
by a moderate turn to the right. Section 3 mimics a straight path. Section 4 reveals a tight turn to the left, just like section 5. Section 6 is another tight turn to the right after a straightway and finally section 7 shows quick changes in direction.

In these experiments we use different levels of control. When we want to create an environment when the two inputs need to be closer in order to shift the weight from the expert to the novice we simply need to set the boundaries of the sets shown in Figure 2 to tighter limits. The three levels of control we use are the following: Tight control, Moderately loose control and Loose control. Table 1 shows the values associated with each level of control. Each set carries four values, (OL, IL, IR, OR), where OL refers to the outer left boundary, IL to the inner left, IR to the inner right and OR to the outer right.

Table 1. Description of fuzzy sets used in classifying the difference between the expert and the novice inputs

\begin{tabular}{|c|c|c|c|}
\hline & Tight & Moderate & Loose \\
\hline WL & $(-\infty,-\infty,-5,-3)$ & $(-\infty,-\infty,-6,-4)$ & $(-\infty,-\infty,-8,-6)$ \\
\hline SL & $(-5,-3,-2,-1)$ & $(-6,-4,-2,-1)$ & $(-8,-6,-4,-2)$ \\
\hline S & $(-2,-1,1,2)$ & $(-2,-1,1,2)$ & $(-4,-2,2,4)$ \\
\hline SR & $(1,2,3,5)$ & $(1,2,4,6)$ & $(2,4,6,8)$ \\
\hline WR & $(3,5,+\infty,+\infty)$ & $(4,6,+\infty,+\infty)$ & $(6,8,+\infty,+\infty)$ \\
\hline
\end{tabular}

The simulated agent is composed of a central unit that contains sensors. The sensors check the terrain in front of the agent for color. The sensors can either pick up white, which is the background, or black, which is the line. The sensors are arranged on a probe that scans the range [-45, $45]$ in front of the agent at 5-degree intervals. Figure 5 shows an image of the agent that is following a line. The light gray is the body of the agent while the dark gray spots in front of it represent the range of action of the sensors.

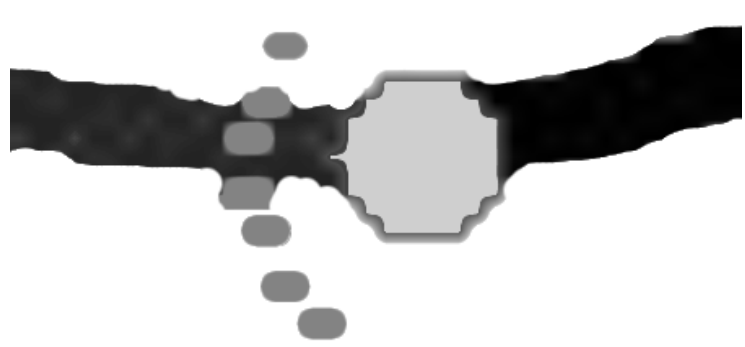

Figure 5. Diagram of the simulated agent with its sensors

The sensors communicate to the agent the color of the terrain at each angle. Then the agent will group together the angles that recorded a reading of a line and will calculate the average. The value calculated will then be analyzed by the agent, which will select the new heading.
An agent can perceive changes in direction up to \pm 45 degrees, given the layout of the sensors.

In order to simulate the behavior of agents we assigned them a preset behavior that allows them to navigate successfully through the pattern selected. In order to simulate an expert agent and a novice one, we chose equations that are slightly different. The typical expert is represented by a simple linear function. When the agent receives the reading from the sensors, the difference in heading is applied directly to the heading, so if the sensors read that, in order to follow the line the agent needs to apply a 15-degree turn to the right, the agent will perform a 15-degree turn to the right.

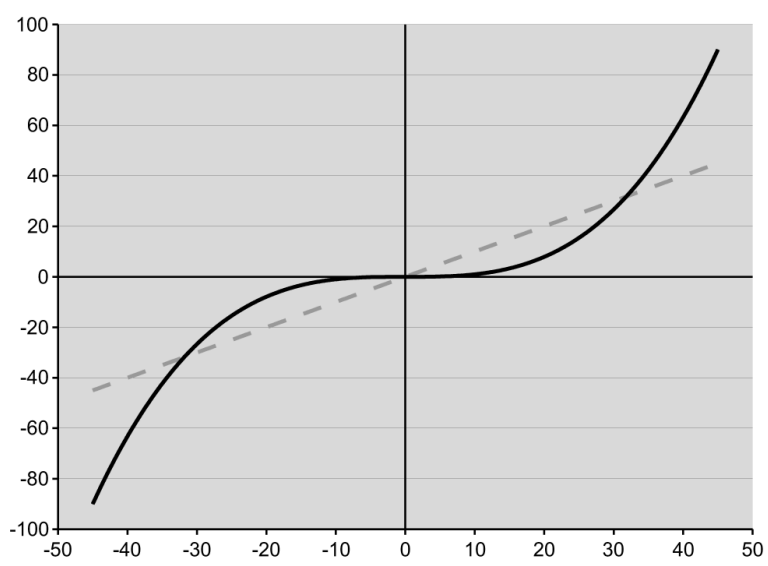

Figure 6. Control functions associated with the expert (dotted) and the novice (solid) controllers

The simulation that represents the case of a novice is powered by an agent that relies on the cube of the difference normalized to the range [-90, 90]. The two equations that are used to drive the agents are reported in Figure 6. The dotted line represents the behavior of the expert and the solid one the behavior of the novice.

Table 2. Controllers used for the simulations

\begin{tabular}{|c|c|c|}
\hline Num & Equation & Simulation \\
\hline 1 & $\mathrm{Y}=\mathrm{X}$ & Expert agent \\
\hline 2 & $\mathrm{Y}=\mathrm{X}^{3}$ & Simple novice agent \\
\hline 3 & $\begin{array}{c}\text { If } \mathrm{X}>0 \text { Then } \\
\mathrm{Y}=\mathrm{X} \\
\text { Else } \\
\mathrm{Y}=0\end{array}$ & $\begin{array}{c}\text { Agent that turns to the right, but } \\
\text { can't turn left. Instead it goes } \\
\text { straight. }\end{array}$ \\
& $\begin{array}{c}\mathrm{X}>0 \text { Then } \\
\mathrm{Y}=\mathrm{X}^{3} \\
\text { Else } \\
4\end{array}$ & $\begin{array}{c}\text { Agent that turns to the right, but } \\
\text { can't turn left. Instead it goes } \\
\text { straight. }\end{array}$ \\
& $\mathrm{Y}=0$ & $\begin{array}{c}\text { Relatively novice agent that only } \\
\text { turns right }\end{array}$ \\
\hline 5 & $\mathrm{Y}=|\mathrm{X}|$ & Novice agent that only turns right \\
\hline 6 & $\mathrm{Y}=\left|\mathrm{X}^{3}\right|$ & \\
\hline
\end{tabular}

This interpretation of the agents shows an expert that acts as expected, with a linear response to the situation. The novice instead reacts more slowly only to overcompensate as the deviation required in order to remain on track 
increases. We also carry out other simulations where the difference between the inputs of the expert and the one of the novice are very different. The equations reported in Table 2 show the driving engines we used.

\section{Experiments}

The first experiment we performed involves an agent navigating through the pattern using fuzzy mediation to blend the controls of the expert powered by controller 1 and the novice simulated by controller 2 . The elements we studied were the differences in behavior of the agent based on different levels of control, as described above. The element we monitored is the value of the mediator's weight. Table 3 shows the average value for the three levels of control using the very same controllers to simulate the controllers' inputs.

Table 3. Agent performance for one lap using different control levels

\begin{tabular}{|c|c|c|c|}
\hline & $\begin{array}{c}\text { Tight } \\
\text { control }\end{array}$ & $\begin{array}{c}\text { Moderate } \\
\text { control }\end{array}$ & $\begin{array}{c}\text { Loose } \\
\text { control }\end{array}$ \\
\hline $\begin{array}{c}\text { Average } \\
\text { mediation } \\
\text { weight }\end{array}$ & 0.59 & 0.62 & 0.82 \\
\hline $\begin{array}{c}\text { Average } \\
\text { difference } \\
\text { between inputs }\end{array}$ & 2.4 & 2.56 & 4.21 \\
\hline $\begin{array}{c}\text { Average } \\
\text { difference } \\
\text { between } \\
\text { expected and } \\
\text { actual path }\end{array}$ & 1.73 & 1.9 & 3.74 \\
\hline
\end{tabular}

We can see that a tighter control reports an average weight of the controller that is lower when compared to the other indices. This means that, when we use the sets that correspond to a tight control of the novice's inputs, the expert retains control for more sections of the patters than in simulations performed using the moderately loose or loose controls.

Table 3 also reports two more values that are important to analyze. The first is the average difference between inputs, or the expert's input and the novice's. The next element of interest is the average difference between the expected and the actual path, measured by the difference between the expert's input and the mediated output. As we can observe from these values, the difference between the expected and the actual path is always smaller than the average difference between the trainer and the trainee's inputs. This shows that fuzzy mediation is successfully mediating between the two controllers, by letting the agent stay on track, and it is also reducing the error that would have been present if this algorithm was not in place.

It is important to note that the values that define the averages between the expert and the novice controllers are always different due to the fact that, with looser control, the agent finds itself further away from the track. The averages reflect the fact that the expert controller wants to over-steer in order to return to the path to follow, while the overall agent does not.

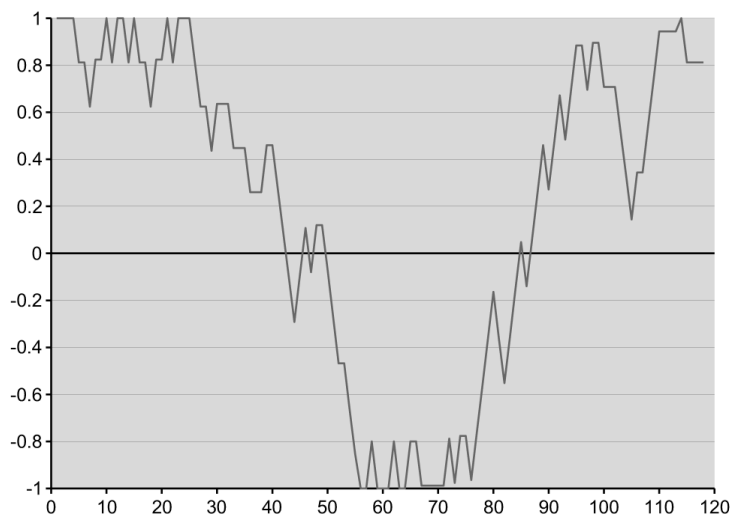

Figure 7. Shift in mediator weight during section 2 of the simulation with tight control

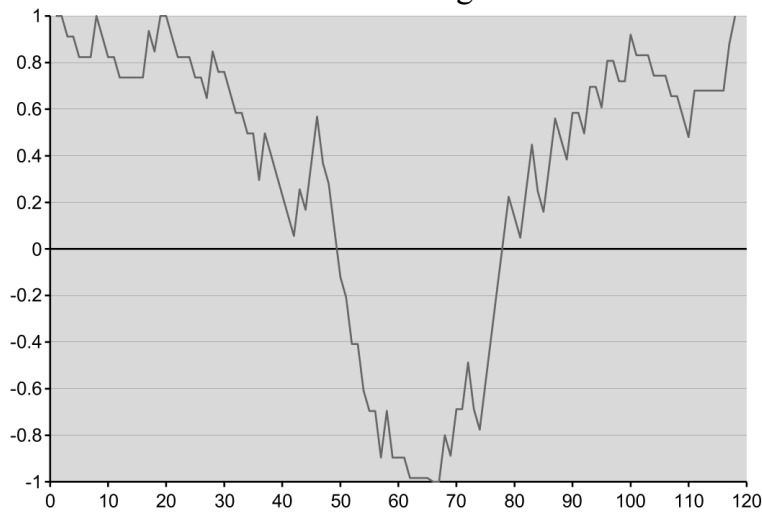

Figure 8. Shift in mediator weight during section 2 of the simulation with moderate control

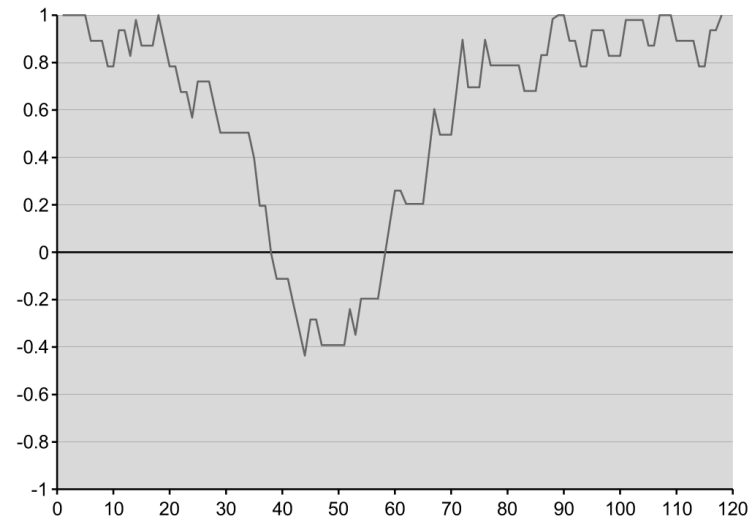

Figure 9. Shift in mediator weight during section 2 of the simulation with loose control

In all the experimental runs we noticed that the area that consistently showed the most shift in control was number 2 in Figure 4. Figures 7, 8 and 9 show the shift in control weight as the agent goes through the turn. The $\mathrm{X}$-axis identifies the number of steps included in the analysis, while the $\mathrm{Y}$-axis identifies the range of the possible weight of the controller's mediation. It is important to note that the agent was following the pattern in a clockwise motion. 
These figures show that, in the case of a tight control, the expert will regain significantly the control of the agent and will perform the steeper section of the turn, as shown in Figure 7. Figure 8 instead shows that the fuzzy mediation that uses a moderately loose control still allows the expert to retain quite a bit of control, but overall the input of the novice is evaluated with a higher importance. Finally, Figure 9 shows that a loose control allows the novice to take care of the majority of the control in this situation. The different nature of the controllers, as described earlier, does not allow the novice to be in control through the entire turn, as the difference in controllers' inputs are quite different.

Table 4. Agent performance in section 2 of the simulation

\begin{tabular}{|c|c|c|c|}
\hline & $\begin{array}{c}\text { Tight } \\
\text { control }\end{array}$ & $\begin{array}{c}\text { Moderate } \\
\text { control }\end{array}$ & $\begin{array}{c}\text { Loose } \\
\text { control }\end{array}$ \\
\hline $\begin{array}{c}\text { Average } \\
\text { mediation } \\
\text { weight }\end{array}$ & 0.21 & 0.31 & 0.57 \\
\hline $\begin{array}{c}\text { Average } \\
\text { difference } \\
\text { between inputs }\end{array}$ & 2.62 & 3.11 & 4.83 \\
\hline $\begin{array}{c}\text { Average } \\
\text { difference } \\
\text { between } \\
\text { expected and } \\
\text { actual path }\end{array}$ & 1.41 & 1.91 & 3.72 \\
\hline
\end{tabular}

Table 4 shows the average weight of the controller's mediator weight for the values plotted in Figures 7, 8 and 9 , showing the control over section 2 of the track.

As we review the performance of the agent over this section of the track, it is important to note that, even in this case, the difference between the inputs of the two controllers is greater than the difference of the expected and the actual output of the agent.

The following experiments were performed in order to study the interaction between an expert and a novice controller when the novice behaves quite differently from the expert. This was done in an effort to study the behavior of the fuzzy mediation architecture when placed in an environment where the novice controller acts in a manner that is radically different from the expert user.

The first simulation was performed with the expert controller based on controller 1 , while controller 3 powered the novice. In the case of a turn to the right the agent showed no problems. We recorded that in this case the agent was leaving the pattern completely in the case of a slight turn to the left. In the case of a sharp turn to the left the agent showed some problems at the beginning of the turn, but no problem after that. This is probably due to the fact that, in the case of a slight deviation control stays unaltered, thus leaving control for a longer period of time to the novice. The second setting involved a novice controller powered by controller 4. This simulation performed very similarly to the previous one, since both simulations for the novice controller show the same response to a left turn.

The third set of experiments focused on controllers that behaved completely different in the case of a left turn. In order to simulate this situation we used controllers 5 and 6 to simulate the novice controllers. We were unable to record extensive data for these experiments because the agent was not able to complete a full loop of the pattern. Our observations show that in the case of controller 5 the agent would initially follow the line, but at the first left turn it would lose control, turn completely around and then get stuck looping around itself. The same behavior was observed when controller 6 powered the novice. We then increased the value by which the weight value of the control is shifted from 0.2 to 0.5 At this point the agent with the novice controller with controller 6 performed almost a full trip around the pattern. It showed problems when it was presented with a slight turn to the left. At that point it would also start looping around itself.

The findings reported in the experiments that used novices powered by controllers 3 through 6 indicate the need to explore thoroughly fuzzy mediation environments that use different increments in the shift of mediation. We performed several other experiments that dealt with the shift in control, not included in this work.

\section{Possible applications}

The possible applications of this research are quite varied, given the possible applications in training, stressing those that involve machinery. This system may be used as a training environment as well as a monitoring one. Several types of machines require "check rides", where the user needs to perform certain operations under scrutiny of evaluators. If the operator fails to perform a certain number of tasks correctly, the license to operate that type of machine will be revoked. With this framework, the operator and the check ride officer may use this approach for evaluation. We may even push the technology a bit further, and allow the operator to be checked by a machine, supervising the operator's performance. Moreover this framework will create a system that learns from the driver's everyday performance. Upon reaching a certain level of confidence for the "autopilot" system, this system will then become the monitor when the driver seems to be impaired in any way and correct the actions of the driver. Should the actions of the driver lead to dangerous driving, the car may calculate the best route to stop and do so to ensure the safety of the passengers as well as other drivers.

Such framework will be extensible to an embedded system that will allow an autopilot type of controller to take over when the machine (automobile/airplane) is capable of producing a path of motion similar to the one followed by the human operator. Such autopilot will also be able to learn from the human controller, so that, the next time similar conditions arise, the machine will be able to foresee the path required to avoid the problem. 
This research will be extensible to other fields, such as the creation of an autopilot system that uses "drives" as parameters to control an automated robot, as explained in [16]. Such robot will also be able to detect and avoid obstacles in an unfriendly environment using the structure described in [17]. Many of the applications within this section are highly dependent on elements that are not in place yet. For example, a digital system that can drive a car efficiently within a regular urban environment is not existent. The authors envision this happening within a few years though, and believe that, when the times are ready, this research will show its true power in its applications. In the mean time, we will create simulations that will allow us to recreate some of the high-end sensors still unavailable to us.

\section{Conclusions and future work}

This article introduced the concept of fuzzy mediation, an innovative and dynamic branch of the field of information fusion. The simulations that were reported in this work show the potential of this algorithm when applied to an agent controlled by two different computational engines. We also identified areas that become of concern as the difference between the controllers increases. We already started experimenting with different solutions for transition strategies between controllers, and the results seem very promising.

\section{References}

[1] Don Norman, There's an Automobile in HCI's Future, Interactions, No. Nov/Dec, pp. 53-54, 2005.

[2] Penelope Grenoble O'Malley, Troubleshooting Machine Control: Contractors Talk About Making the Most of $3 D$ GPS, Grading \& Excavator Contractor, Vol. 2, No. 7, 2005.

[3] James Wallace, Unlike Airbus, Boeing lets aviator override fly-by-wire technology, Seattle Post-Intelligencer, March 20, 2000.

[4] WordNET Search, Control, Retrieved February 16th, 2006 from http://wordnet.princeton.edu/perl/webwn? $\mathrm{s}=$ control.

[5] Mieczyslaw Kokar, Jerzy Tomasik and Jerzy Weyman, Formalizing Classes of Information Fusion Systems, Information Fusion, Vol. 3, No. 5, pp. 189-202, 2004.

[6] Goran Trajkovski, E-POPSICLE: An Online Environment for Studying Context Learning in Human and Artificial Agents. $16^{\text {th }}$ Midwest AI and Cognitive Science Conference, 2005, Proceedings, pp. 61-66.
[7] Pedro Abad et al., Using Supervised Learning Techniques for Diagnosis of Dynamic Systems, International Workshop on Principles of Diagnosis, 2002, Proceedings.

[8] Anuradha Gokhale, Collaborative Learning Enhances Critical Thinking. Journal of Technology Education, Vol. 1, No. 7, pp. 22-30, 2005.

[9] Keith Hammonds et al., The New University - A Tough Market is Reshaping Colleges, Business Week, No. 22, pp. 96-102, 1997.

[10] Bob Dugan and Ephraim Glinert, Task Division in Collaborative Simulations, $35^{\text {th }}$ Annual Hawaii International Conference on System Sciences, 2002, Proceedings.

[11] Stephen Kwek, An Apprentice Learning Model, $12^{\text {th }}$ annual conference on Computational learning theory, 1999, Proceedings, pp. 63-74.

[12] Sachiyo Arai and Toru Ishida, Learning for HumanAgent Collaboration on the Semantic Web, 12th International Conference on Informatics Research for Development of Knowledge Society Infrastructure, 2004, Proceedings, pp. 132-139.

[13] Goran Trajkovski, An Imitation-Based Approach to Modeling Homogenous Agents Societies, IDEA Publishing, Hershey, PA, 2007.

[14] Giovanni Vincenti and Goran Trajkovski, Fuzzy Mediation for Online Learning in Autonomous Agents, 2006 Fall AAAI Symposium, Arlington, VA. USA, October 12-15 2006, pp. 127-133.

[15] Zadeh, Lotfi, Fuzzy Sets, Information and Control, No. 8, pp. 338-353, 1965.

[16] Goran Trajkovski et al., Building infrastructure for an honors research robotics lab, $6^{\text {th }}$ ACIS International Conference on Software Engineering, Artificial Intelligence, Networking, and Parallel/Distributed Computing, Towson, MD, 2005, Proceedings, pp. 352357.

[17] Goran Trajkovski, Georgi Stojanov, and Giovanni Vincenti, Extending MASIVE: The impact of stress on imitation-based learning, $6^{\text {th }}$ ACIS International Conference on Software Engineering, Artificial Intelligence, Networking, and Parallel/Distributed Computing, Towson, MD, 2005, Proceedings, pp. 360365. 